\title{
Parameter-free Sentence Embedding via Orthogonal Basis
}

\author{
Ziyi Yang ${ }^{*}$, Chenguang $\mathrm{Zhu}^{2}$, and Weizhu $\mathrm{Chen}^{3}$ \\ ${ }^{1}$ Department of Mechanical Engineering, Stanford university \\ ${ }^{2}$ Microsoft Speech and Dialogue Research Group \\ ${ }^{3}$ Microsoft Dynamics 365 AI \\ ziyi.yang@stanford.edu, \{chezhu, wzchen\}@microsoft.com
}

\begin{abstract}
We propose a simple and robust nonparameterized approach for building sentence representations. Inspired by the GramSchmidt Process in geometric theory, we build an orthogonal basis of the subspace spanned by a word and its surrounding context in a sentence. We model the semantic meaning of a word in a sentence based on two aspects. One is its relatedness to the word vector subspace already spanned by its contextual words. The other is the word's novel semantic meaning which shall be introduced as a new basis vector perpendicular to this existing subspace. Following this motivation, we develop an innovative method based on orthogonal basis to combine pre-trained word embeddings into sentence representations. This approach requires zero parameters, along with efficient inference performance. We evaluate our approach on 11 downstream NLP tasks. Our model shows superior performance compared with non-parameterized alternatives and it is competitive to other approaches relying on either large amounts of labelled data or prolonged training time.
\end{abstract}

\section{Introduction}

The concept of word embeddings has been prevalent in NLP community in recent years, as they can characterize semantic similarity between any pair of words, achieving promising results in a large number of NLP tasks (Mikolov et al., 2013; Pennington et al., 2014; Salle et al., 2016). However, due to the hierarchical nature of human language, it is not sufficient to comprehend text solely based on isolated understanding of each word. This has prompted a recent rise in search for semantically

\footnotetext{
${ }^{*}$ Most of the work was done during internship at Microsoft

Published as a conference paper at EMNLP 2019 (oral presentation)
}

robust embeddings for longer pieces of text, such as sentences and paragraphs.

Based on learning paradigms, the existing approaches to sentence embeddings can be categorized into two categories: i) parameterized methods and ii) non-parameterized methods.

Parameterized sentence embeddings. These models are parameterized and require training to optimize their parameters. SkipThought (Kiros et al., 2015) is an encoder-decoder model that predicts adjacent sentences. Pagliardini et al. (2018) proposes an unsupervised model, Sent2Vec, to learn an $\mathrm{n}$-gram feature in a sentence to predict the center word from the surrounding context. Quick thoughts (QT) (Logeswaran and Lee, 2018) replaces the encoder with a classifier to predict context sentences from candidate sequences. Khodak et al. (2018) proposes à la carte to learn a linear mapping to reconstruct the center word from its context. Conneau et al. (2017) generates the sentence encoder InferSent using Natural Language Inference (NLI) dataset. Universal Sentence Encoder (Yang et al., 2018; Cer et al., 2018) utilizes the emerging transformer structure (Vaswani et al., 2017; Devlin et al., 2018) that has been proved powerful in various NLP tasks. The model is first trained on large scale of unsupervised data from Wikipedia and forums, and then trained on the Stanford Natural Language Inference (SNLI) dataset. Wieting and Gimpel (2017b) propose the gated recurrent averaging network (GRAN), which is trained on Paraphrase Database (PPDB) and English Wikipedia. Subramanian et al. (2018) leverages a multi-task learning framework to generate sentence embeddings. Wieting et al. (2015a) learns the paraphrastic sentence representations as the simple average of updated word embeddings.

Non-parameterized sentence embedding. Recent work (Arora et al., 2017) shows that, surprisingly, a weighted sum or transformation 
of word representations can outperform many sophisticated neural network structures in sentence embedding tasks. These methods are parameter-free and require no further training upon pre-trained word vectors. Arora et al. (2017) constructs a sentence embedding called SIF as a sum of pre-trained word embeddings, weighted by reverse document frequency. Ethayarajh (2018) builds upon the random walk model proposed in SIF by setting the probability of word generation inversely related to the angular distance between the word and sentence embeddings. Rücklé et al. (2018) concatenates different power mean word embeddings as a sentence vector in $p$-mean. As these methods do not have a parameterized model, they can be easily adapted to novel text domains with both fast inference speed and high-quality sentence embeddings. In view of this trend, our work aims to further advance the frontier of this group and make its new state-of-the-art.

In this paper, we propose a novel sentence embedding algorithm, Geometric Embedding (GEM), based entirely on the geometric structure of word embedding space. Given a $d$ $\operatorname{dim}$ word embedding matrix $\boldsymbol{A} \in \mathbb{R}^{d \times n}$ for a sentence with $n$ words, any linear combination of the sentence's word embeddings lies in the subspace spanned by the $n$ word vectors. We analyze the geometric structure of this subspace in $\mathbb{R}^{d}$. When we consider the words in a sentence one-by-one in order, each word may bring in a novel orthogonal basis to the existing subspace. This new basis can be considered as the new semantic meaning brought in by this word, while the length of projection in this direction can indicate the intensity of this new meaning. It follows that a word with a strong intensity should have a larger influence in the sentence's meaning. Thus, these intensities can be converted into weights to linearly combine all word embeddings to obtain the sentence embedding. In this paper, we theoretically frame the above approach in a QR factorization of the word embedding matrix $\boldsymbol{A}$. Furthermore, since the meaning and importance of a word largely depends on its close neighborhood, we propose the sliding-window QR factorization method to capture the context of a word and characterize its significance within the context.

In the last step, we adapt a similar approach as Arora et al. (2017) to remove top principal vectors before generating the final sentence embed- ding. This step is to ensure commonly shared background components, e.g. stop words, do not bias sentence similarity comparison. As we build a new orthogonal basis for each sentence, we propose to have disparate background components for each sentence. This motivates us to put forward a sentence-specific principal vector removal method, leading to better empirical results.

We evaluate our algorithm on 11 NLP tasks. Our algorithm outperforms all non-parameterized methods and many parameterized approaches in 10 tasks. Compared to SIF (Arora et al., 2017), the performance is boosted by $5.5 \%$ on STS benchmark dataset, and by $2.5 \%$ on SST dataset. Plus, the running time of our model compares favorably with existing models.

The rest of this paper is organized as following. In Section 2, we describe our sentence embedding algorithm GEM. We evaluate our model on various tasks in Section 3 and Section 4. Finally, we summarize our work in Section 5. Our implementation is available online ${ }^{1}$.

\section{Approach}

We introduce three scores to quantify the importance of a word, as will be explained in this section. First, novelty score $\alpha_{n}$ measures the portion of the new semantic meaning in a word. Second, significance score $\alpha_{s}$ describes the alignment between the new semantic meaning and the sentence-level meaning. Finally, uniqueness score $\alpha_{u}$ examines the uniqueness of the new semantic meaning in the corpus level.

\subsection{Quantify New Semantic Meaning}

Let us consider the idea of word embeddings (Mikolov et al., 2013), where a word $w_{i}$ is projected as a vector $\boldsymbol{v}_{w_{i}} \in \mathbb{R}^{d}$. Any sequence of words can be viewed as a subspace in $\mathbb{R}^{d}$ spanned by its word vectors. Before the appearance of the $i$ th word, $\boldsymbol{S}$ is a subspace in $\mathbb{R}^{d}$ spanned by $\left\{\boldsymbol{v}_{w_{1}}, \boldsymbol{v}_{w_{2}}, \ldots, \boldsymbol{v}_{w_{i-1}}\right\}$. Its orthonormal basis is $\left\{\boldsymbol{q}_{1}, \boldsymbol{q}_{2}, \ldots, \boldsymbol{q}_{i-1}\right\}$. The embedding $\boldsymbol{v}_{w_{i}}$ of the $i$ th

\footnotetext{
${ }^{1}$ https://github.com/ziyi-yang/GEM
} 
word $w_{i}$ can be decomposed into

$$
\begin{aligned}
\boldsymbol{v}_{w_{i}} & =\sum_{j=1}^{i-1} r_{j} \boldsymbol{q}_{j}+r_{i} \boldsymbol{q}_{i} \\
r_{j} & =\boldsymbol{q}_{j}^{T} \boldsymbol{v}_{w_{i}} \\
r_{i} & =\left\|\boldsymbol{v}_{w_{i}}-\sum_{j=1}^{i-1} r_{j} \boldsymbol{q}_{j}\right\|_{2}
\end{aligned}
$$

where $\sum_{j=1}^{i-1} r_{j} \boldsymbol{q}_{j}$ is the part in $\boldsymbol{v}_{w_{i}}$ that resides in subspace $\boldsymbol{S}$, and $\boldsymbol{q}_{i}$ is orthogonal to $\boldsymbol{S}$ and is to be added to $\boldsymbol{S}$. The above algorithm is also known as Gram-Schmidt Process. In the case of rank deficiency, i.e., $\boldsymbol{v}_{w_{i}}$ is already a linear combination of $\left\{\boldsymbol{q}_{1}, \boldsymbol{q}_{2}, \ldots \boldsymbol{q}_{i-1}\right\}, \boldsymbol{q}_{i}$ is a zero vector and $r_{i}=0$. In matrix form, this process is also known as $Q R$ factorization, defined as follows.

QR factorization. Define an embedding matrix of $n$ words as $\boldsymbol{A}=\left[\boldsymbol{A}_{:, 1}, \boldsymbol{A}_{:, 2}, \ldots, \boldsymbol{A}_{:, n}\right] \in \mathbb{R}^{d \times n}$, where $\boldsymbol{A}_{:, i}$ is the embedding of the $i$ th word $w_{i}$ in a word sequence $\left(w_{1}, \ldots, w_{i}, \ldots, w_{n}\right) . \boldsymbol{A} \in \mathbb{R}^{d \times n}$ can be factorized into $\boldsymbol{A}=\boldsymbol{Q R}$, where the nonzero columns in $Q \in \mathbb{R}^{d \times n}$ are the orthonormal basis, and $\boldsymbol{R} \in \mathbb{R}^{n \times n}$ is an upper triangular matrix.

The process above computes the novel semantic meaning of a word w.r.t all preceding words. As the meaning of a word influences and is influenced by its close neighbors, we now calculate the novel orthogonal basis vector $\boldsymbol{q}_{i}$ of each word $w_{i}$ in its neighborhood, rather than only w.r.t the preceding words.

\section{Definition 1 (Contextual Window Matrix)}

Given $a$ word $w_{i}$, and its $m$ neighborhood window inside the sentence $\left(w_{i-m}, \ldots, w_{i-1}, w_{i}, w_{i+1}, \ldots, w_{i+m}\right)$, define the contextual window matrix of word $w_{i}$ as:

$$
\boldsymbol{S}^{i}=\left[\boldsymbol{v}_{w_{i-m}} \ldots \boldsymbol{v}_{w_{i-1}}, \boldsymbol{v}_{w_{i+1}} \ldots \boldsymbol{v}_{w_{i+m}}, \boldsymbol{v}_{w_{i}}\right]
$$

Here we shuffle $\boldsymbol{v}_{w_{i}}$ to the end of $\boldsymbol{S}^{i}$ to compute its novel semantic information compared with its context. Now the QR factorization of $\boldsymbol{S}^{i}$ is

$$
\boldsymbol{S}^{i}=\boldsymbol{Q}^{i} \boldsymbol{R}^{i}
$$

Note that $\boldsymbol{q}_{i}$ is the last column of $\boldsymbol{Q}^{i}$, which is also the new orthogonal basis vector to this contextual window matrix.

Next, in order to generate the embedding for a sentence, we will assign a weight to each of its words. This weight should characterize how much new and important information a word brings to the sentence. The previous process yields the orthogonal basis vector $\boldsymbol{q}_{i}$. We propose that $\boldsymbol{q}_{i}$ represents the novel semantic meaning brought by word $w_{i}$. We will now discuss how to quantify i) the novelty of $\boldsymbol{q}_{i}$ to other meanings in $w_{i}$, ii) the significance of $\boldsymbol{q}_{i}$ to its context, and iii) the corpuswise uniqueness of $\boldsymbol{q}_{i}$ w.r.t the whole corpus.

\subsection{Novelty}

We propose that a word $w_{i}$ is more important to a sentence if its novel orthogonal basis vector $\boldsymbol{q}_{i}$ is a large component in $\boldsymbol{v}_{w_{i}}$, quantified by the proposed novelty score $\alpha_{n}$. Let $\boldsymbol{r}$ denote the last column of $\boldsymbol{R}^{i}$, and $\boldsymbol{r}_{-1}$ denote the last element of $\boldsymbol{r}$, $\alpha_{n}$ is defined as:

$$
\alpha_{n}=\exp \left(\frac{\left\|\boldsymbol{q}_{i}\right\|_{2}}{\left\|\boldsymbol{v}_{w_{i}}\right\|_{2}}\right)=\exp \left(\frac{\boldsymbol{r}_{-1}}{\|\boldsymbol{r}\|_{2}}\right)
$$

Note that $\left\|\boldsymbol{q}_{i}\right\|_{2}=\boldsymbol{r}_{-1}$ and $\left\|\boldsymbol{v}_{w_{i}}\right\|_{2}=\|\boldsymbol{r}\|_{2}$. One can show that $\alpha_{n}$ is the exponential of the normalized distance between $\boldsymbol{v}_{w_{i}}$ and the subspace spanned by its context.

\subsection{Significance}

The significance of a word is related to how semantically aligned it is to the meaning of its context. To identify principal directions, i.e. meanings, in the contextual window matrix $\boldsymbol{S}^{i}$, we employ Singular Value Decomposition.

Singular Value Decomposition. Given a matrix $\boldsymbol{A} \in \mathbb{R}^{d \times n}$, there exists $\boldsymbol{U} \in \mathbb{R}^{d \times n}$ with orthogonal columns, diagonal matrix $\boldsymbol{\Sigma}=$ $\operatorname{diag}\left(\sigma_{1}, \ldots, \sigma_{n}\right), \sigma_{1} \geq \sigma_{2} \geq \ldots \geq \sigma_{n} \geq 0$, and orthogonal matrix $\boldsymbol{V} \in \mathbb{R}^{n \times n}$, such that $\boldsymbol{A}=$ $\boldsymbol{U} \boldsymbol{\Sigma} \boldsymbol{V}^{T}$.

The columns of $\boldsymbol{U},\left\{\boldsymbol{U}_{:, j}\right\}_{j=1}^{n}$, are an orthonormal basis of $\boldsymbol{A}$ 's columns subspace and we propose that they represent a set of semantic meanings from the context. Their corresponding singular values $\left\{\sigma_{j}\right\}_{j=1}^{n}$, denoted by $\sigma(\boldsymbol{A})$, represent the importance associated with $\left\{\boldsymbol{U}_{:, j}\right\}_{j=1}^{n}$. The SVD of $w_{i}$ 's contextual window matrix is $\boldsymbol{S}^{i}=$ $\boldsymbol{U}^{i} \boldsymbol{\Sigma}^{i} \boldsymbol{V}^{i T} \in \mathbb{R}^{d \times(2 m+1)}$. It follows that $\boldsymbol{q}_{i}^{T} \boldsymbol{U}^{i}$ is the coordinate of $\boldsymbol{q}_{i}$ in the basis of $\left\{\boldsymbol{U}_{:, j}^{i}\right\}_{j=1}^{2 m+1}$.

Intuitively, a word is more important if its novel semantic meaning has a better alignment with more principal meanings in its contextual window. This can be quantified as $\left\|\sigma\left(\boldsymbol{S}^{i}\right) \odot\left(\boldsymbol{q}_{i}^{T} \boldsymbol{U}^{i}\right)\right\|_{2}$, where $\odot$ denotes element-wise product. Therefore, we define the significance of $w_{i}$ in its context 
to be:

$$
\alpha_{s}=\frac{\left\|\sigma\left(\boldsymbol{S}^{i}\right) \odot\left(\boldsymbol{q}_{i}^{T} \boldsymbol{U}^{i}\right)\right\|_{2}}{2 m+1}
$$

It turns out $\alpha_{s}$ can be rewritten as

$$
\begin{aligned}
\alpha_{s} & =\frac{\left\|\boldsymbol{q}_{i}^{T} \boldsymbol{U}^{i} \boldsymbol{\Sigma}^{i}\right\|_{2}}{2 m+1}=\frac{\left\|\boldsymbol{q}_{i}^{T} \boldsymbol{U}^{i} \boldsymbol{\Sigma}^{i} \boldsymbol{V}^{i}\right\|_{2}}{2 m+1} \\
& =\frac{\left\|\boldsymbol{q}_{i}^{T} \boldsymbol{S}^{i}\right\|_{2}}{2 m+1}=\frac{\boldsymbol{q}_{i}^{T} \boldsymbol{v}_{w_{i}}}{2 m+1}=\frac{\boldsymbol{r}_{-1}}{2 m+1}
\end{aligned}
$$

and we use the fact that $\boldsymbol{V}^{i}$ is an orthogonal matrix and $\boldsymbol{q}_{i}$ is orthogonal to all but the last column of $\boldsymbol{S}^{i}, \boldsymbol{v}_{w_{i}}$. Therefore, $\alpha_{s}$ is essentially the distance between $\boldsymbol{w}_{i}$ and the context hyper-plane, normalized by the context size.

Although $\alpha_{s}$ and $\alpha_{n}$ look alike in mathematics form, they model distinct quantities in word $w_{i}$ against its contextual window. $\alpha_{n}$ is a function of $\left\|\boldsymbol{q}_{i}\right\|_{2}$ divided by $\left\|\boldsymbol{w}_{i}\right\|_{2}$, i.e., the portion of the new semantic meaning in word $w_{i}$. In contrast, eq. (6) shows that $\alpha_{s}$ equals $\left\|\boldsymbol{q}_{i}\right\|_{2}$ divided by a constant, namely $\alpha_{s}$ quantifies the absolute magnitude of the new semantic meaning $\boldsymbol{q}_{i}$.

\subsection{Corpus-wise Uniqueness}

Similar to the idea of inverse document frequency (IDF) (Sparck Jones, 1972), a word that is commonly present in the corpus is likely to be a stop word, thus its corpus-wise uniqueness is small. In our solution, we compute the principal directions of the corpus and then measure their alignment with the novel orthogonal basis vector $\boldsymbol{q}_{i}$. If there is a high alignment, $w_{i}$ will be assigned a relatively low corpus-wise uniqueness score, and vice versa.

\subsubsection{Compute Principal Directions of Corpus}

In Arora et al. (2017), given a corpus containing a set of $N$ sentences, an embedding matrix $\boldsymbol{X}=\left[\boldsymbol{x}_{1}, \boldsymbol{x}_{2}, \ldots, \boldsymbol{x}_{N}\right] \in \mathbb{R}^{d \times N}$ is generated, where $\boldsymbol{x}_{i}$ is the sentence embedding for the $i$-th sentence in the corpus, computed by SIF algorithm. Then principal vectors of $\boldsymbol{X}$ are computed and projections onto the principal vectors are removed from each sentence embedding $\boldsymbol{x}_{i}$.

In contrast to Arora et al. (2017), we do not form the embedding matrix after we obtain the final sentence representation. Instead, we obtain an intermediate coarse-grained embedding matrix $\boldsymbol{X}^{c}=\left[\boldsymbol{g}_{1}, \ldots, \boldsymbol{g}_{N}\right]$ as follows. Suppose the
SVD of the sentence matrix of the $i$ th sentence is $\boldsymbol{S}=\left[\boldsymbol{v}_{w_{1}}, \ldots, \boldsymbol{v}_{w_{n}}\right]=\boldsymbol{U} \boldsymbol{\Sigma} \boldsymbol{V}^{T}$. Then the coarsegrained embedding for the $i$ th sentence is defined as:

$$
\boldsymbol{g}_{i}=\sum_{j=1}^{n} f\left(\sigma_{j}\right) \boldsymbol{U}_{:, j}
$$

where $f\left(\sigma_{j}\right)$ is a monotonically increasing function. We then compute the top $K$ principal vectors $\left\{\boldsymbol{d}_{1}, \ldots, \boldsymbol{d}_{K}\right\}$ of $\boldsymbol{X}^{c}$, with singular values $\sigma_{1} \geq$ $\sigma_{2} \geq \ldots \geq \sigma_{K}$.

\subsubsection{Uniqueness Score}

In contrast to Arora et al. (2017), we select different principal vectors of $\boldsymbol{X}^{c}$ for each sentence, as different sentences may have disparate alignments with the corpus. For each sentence, $\left\{\boldsymbol{d}_{1}, \ldots, \boldsymbol{d}_{K}\right\}$ are re-ranked in descending order of their correlation with sentence matrix $\boldsymbol{S}$. The correlation is defined as:

$$
o_{i}=\sigma_{i}\left\|\boldsymbol{S}^{T} \boldsymbol{d}_{i}\right\|_{2}, 1 \leq i \leq K
$$

Next, the top $h$ principal vectors after reranking based on $o_{i}$ are selected: $\boldsymbol{D}=$ $\left\{\boldsymbol{d}_{t_{1}}, \ldots, \boldsymbol{d}_{t_{h}}\right\}$, with $o_{t_{1}} \geq o_{t_{2}} \geq \ldots \geq o_{t_{h}}$ and their singular values in $\boldsymbol{X}^{c}$ are $\boldsymbol{\sigma}_{d}=\left[\sigma_{t_{1}}, \ldots, \sigma_{t_{h}}\right] \in$ $\mathbb{R}^{h}$.

Finally, a word $w_{i}$ with new semantic meaning vector $\boldsymbol{q}_{i}$ in this sentence will be assigned a corpus-wise uniqueness score:

$$
\alpha_{u}=\exp \left(-\left\|\boldsymbol{\sigma}_{d} \odot\left(\boldsymbol{q}_{i}^{T} \boldsymbol{D}\right)\right\|_{2} / h\right)
$$

This ensures that common stop words will have their effect diminished since their embeddings are closely aligned with the corpus' principal directions.

\subsection{Sentence Vector}

A sentence vector $\boldsymbol{c}_{s}$ is computed as a weighted sum of its word embeddings, where the weights come from three scores: a novelty score $\left(\alpha_{n}\right)$, a significance score $\left(\alpha_{s}\right)$ and a corpus-wise uniqueness score $\left(\alpha_{u}\right)$.

$$
\begin{aligned}
& \alpha_{i}=\alpha_{n}+\alpha_{s}+\alpha_{u} \\
& \boldsymbol{c}_{s}=\sum_{i} \alpha_{i} \boldsymbol{v}_{w_{i}}
\end{aligned}
$$

We provide a theoretical explanation of Equation (10) in Appendix. 
Sentence-Dependent Removal of Principal Components. Arora et al. (2017) shows that given a set of sentence vectors, removing projections onto the principal components of the spanned subspace can significantly enhance the performance on semantic similarity task. However, as each sentence may have a different semantic meaning, it could be sub-optimal to remove the same set of principal components from all sentences.

Therefore, we propose the sentence-dependent principal component removal (SDR), where we re-rank top principal vectors based on correlation with each sentence. Using the method from Section 2.4.2, we obtain $\boldsymbol{D}=\left\{\boldsymbol{d}_{t_{1}}, \ldots, \boldsymbol{d}_{t_{r}}\right\}$ for a sentence $s$. The final embedding of this sentence is then computed as:

$$
\boldsymbol{c}_{s} \leftarrow \boldsymbol{c}_{s}-\sum_{j=1}^{r}\left(\boldsymbol{d}_{t_{j}}^{T} \boldsymbol{c}_{s}\right) \boldsymbol{d}_{t_{j}}
$$

Ablation experiments show that sentencedependent principal component removal can achieve better result. The complete algorithm is summarized in Algorithm 1 with an illustration in Figure 1.

\subsection{Handling of out-of-vocabulary Words}

In many NLP algorithms, the out-of-vocabulary (OOV) words are projected to a special "UNK" token. However, in this way, different OOV words with drastically different meanings will share the same embedding. To fix this problem, we change this projection method by mapping OOVs to pretrained in-vocabulary words, based on a hash function SHA-256 of its characters. In this way, two different OOV words will almost certainly have different embeddings. In the experiments, we apply this OOV projection technique in both STS-B and CQA tasks.

\section{Experiments}

\subsection{Semantic Similarity Tasks: STS Benchmark}

We evaluate our model on the STS Benchmark (Cer et al., 2017), a sentence-level semantic similarity dataset. The goal for a model is to predict a similarity score of two sentences given a sentence pair. The evaluation is by the Pearson's coefficient $r$ between human-labeled similarity ( $0-5$ points $)$ and predictions.

Experimental settings. We report two versions of our model, one only using GloVe word vectors

\begin{tabular}{ccc}
\hline Non-parameterized models & dev & test \\
\hline GEM + L.F.P (ours) & 83.5 & 78.4 \\
GEM + LexVec (ours) & 81.9 & 76.5 \\
SIF (Arora et al., 2017) & 80.1 & 72.0 \\
uSIF (Ethayarajh, 2018) & $\mathbf{8 4 . 2}$ & 79.5 \\
LexVec & 58.78 & 50.43 \\
L.F.P & 62.4 & 52.0 \\
word2vec skipgram & 70.0 & 56.5 \\
Glove & 52.4 & 40.6 \\
ELMo & 64.6 & 55.9 \\
\hline Parameterized models & & \\
\hline PARANMT-50M (Wieting and Gimpel, 2017a) & - & $\mathbf{7 9 . 9}$ \\
Reddit + SNLI (Yang et al., 2018) & 81.4 & 78.2 \\
GRAN (Wieting and Gimpel, 2017b) & 81.8 & 76.4 \\
InferSent (Conneau et al., 2017) & 80.1 & 75.8 \\
Sent2Vec (Pagliardini et al., 2018) & 78.7 & 75.5 \\
Paragram-Phrase (Wieting et al., 2015a) & 73.9 & 73.2 \\
\hline
\end{tabular}

Table 1: Pearson's $r \times 100$ on STSB. Best results are in bold.

\begin{tabular}{cc}
\hline GEM + L.F.P (ours) & $\mathbf{4 9 . 1 1}$ \\
Reddit + SNLI tuned & 47.44 \\
\hline KeLP-contrastive1 & 49.00 \\
SimBow-contrastive2 & 47.87 \\
SimBow-primary & 47.22 \\
\hline
\end{tabular}

Table 2: MAP on CQA subtask B.

(GEM + GloVe), and the other using word vectors concatenated from Lex Vec, fastText and PSL (Wieting et al., 2015b) (GEM + L.F.P). The final similarity score is computed as an inner product of normalized sentence vectors. Since our model is non-parameterized, it does not utilize any information from the dev set when evaluating on the test set and vice versa. Hyper-parameters are chosen at $m=7, h=17, K=45$, and $t=3$ by conducing hyper-parameters search on dev set. Results on the dev and test set are reported in Table 1. As shown, on the test set, our model has a $6.4 \%$ higher score compared with another non-parameterized model SIF, and $26.4 \%$ higher than the baseline of averaging L.F.P word vectors. It also outperforms all parameterized models including GRAN, InferSent, Sent2Vec and Reddit+SNLI.

\subsection{Semantic Similarity Tasks: CQA}

We evaluate our model on subtask B of the SemEval Community Question Answering (CQA) task, another semantic similarity dataset. Given an original question $Q_{o}$ and a set of the first ten related questions $\left(Q_{1}, \ldots, Q_{10}\right)$ retrieved by a search engine, the model is expected to re-rank the related 


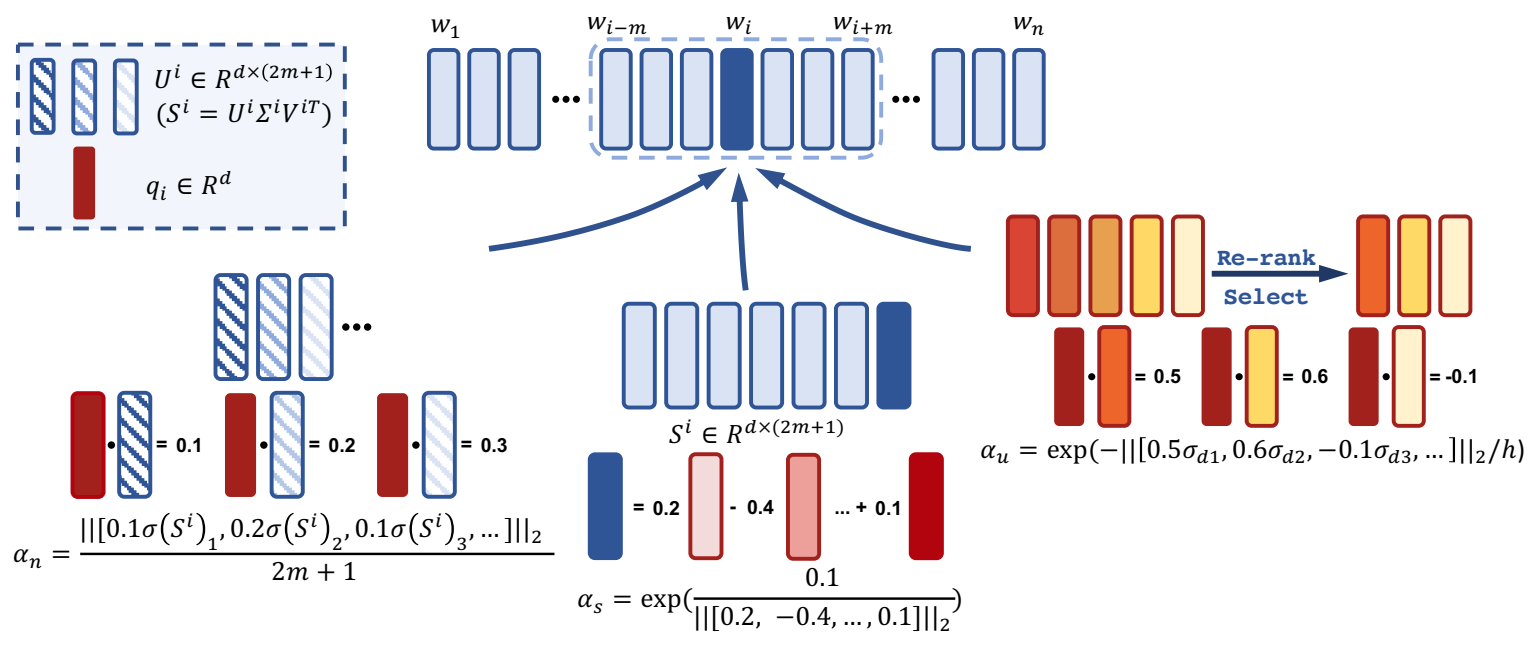

Figure 1: An illustration of GEM algorithm. Top middle: The sentence to encode, with words $w_{1}$ to $w_{n}$. The contextual window of word $w_{i}$ is inside the dashed line. Bottom middle: Form contextual window matrix $\boldsymbol{S}^{i}$ for $w^{i}$, compute $\boldsymbol{q}_{i}$ and novelty score $\alpha_{n}$ (Section 2.1 and Section 2.2). Bottom left: SVD of $\boldsymbol{S}^{i}$ and compute the significance score $\alpha_{s}$ (Section 2.3). Bottom right: Re-rank and select from principal components (orange blocks) and compute uniqueness score $\alpha_{u}$ (Section 2.4).

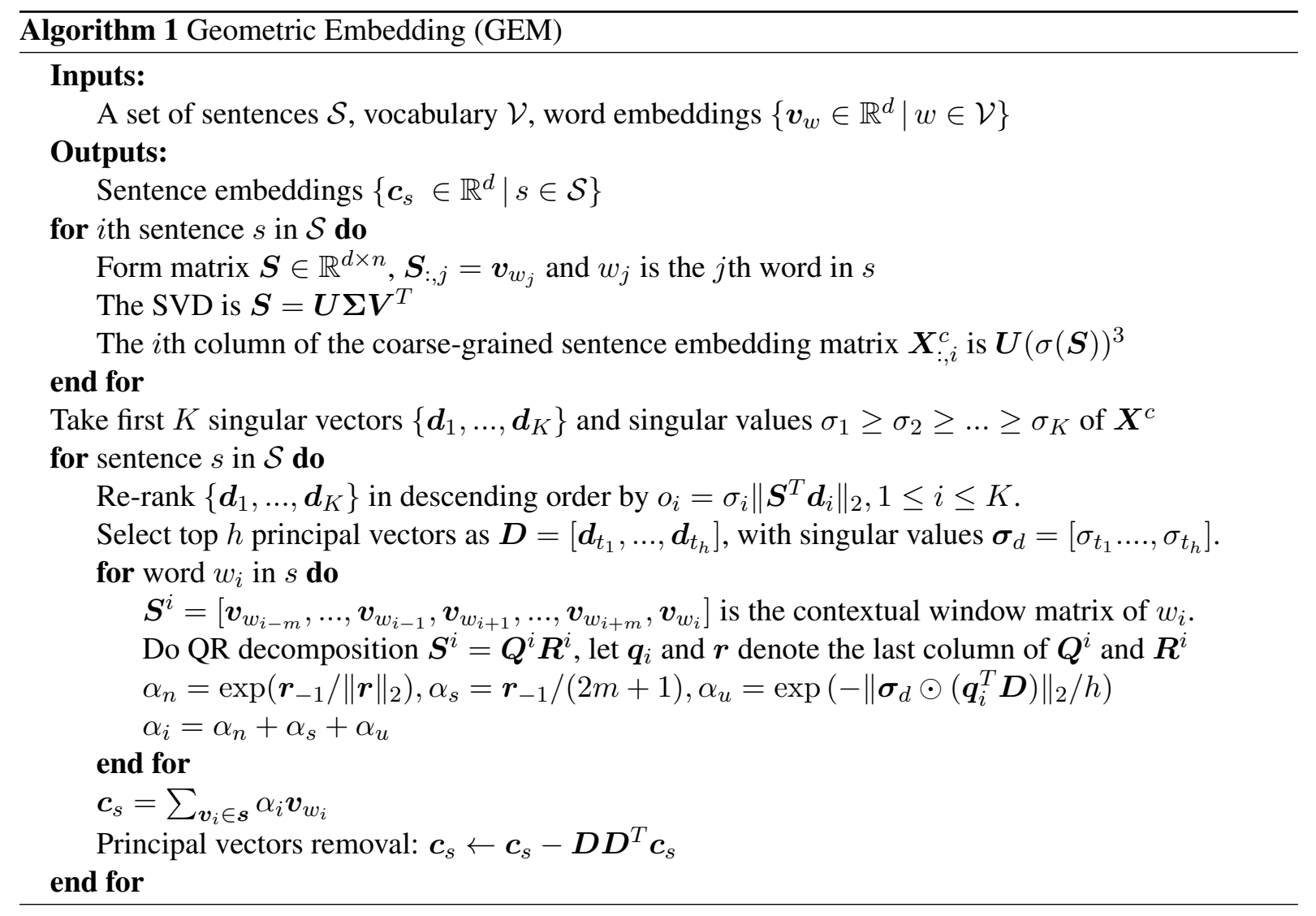

questions according to their similarity with respect to the original question. Each retrieved question $Q_{i}$ is labelled "PerfectMatch", "Relevant" or "Irrelevant", with respect to $Q_{o}$. Mean average precision (MAP) is used as the evaluation measure.

We encode each question text into a unit vector $\boldsymbol{u}$. Retrieved questions $\left\{Q_{i}\right\}_{i=1}^{10}$ are ranked according to their cosine similarity with $Q_{o}$. Results are shown in Table 2. For comparison, we include results from the best models in 2017 competition: SimBow (Charlet and Damnati, 2017), KeLP (Filice et al., 2017), and Reddit + SNLI tuned. Note that all three benchmark models require learning on CQA training set, and SimBow 
and KeLP leverage optional features including usage of comments and user profiles. In comparison, our model only uses the question text without any training. Our model clearly outperforms Reddit + SNLI tuned, SimBow-primary and KeLP model.

\subsection{Supervised tasks}

We further test our model on nine supervised tasks, including seven classification tasks: movie review (MR) (Pang and Lee, 2005), Stanford Sentiment Treebank (SST) (Socher et al., 2013), question-type classification (TREC) (Voorhees and Dang, 2003), opinion polarity (MPQA) (Wiebe et al., 2005), product reviews (CR) (Hu and Liu, 2004), subjectivity/objectivity classification (SUBJ) (Pang and Lee, 2004) and paraphrase identification (MRPC) (Dolan et al., 2004). We also evaluate on two entailment and semantic relatedness tasks: SICK similarity (SICK-R) and the SICK entailment (SICK-E) (Marelli et al., 2014). The sentence embeddings generated are fixed and only the downstream task-specific neural structure is learned. For classification tasks, a linear classifier is trained on top, following Kiros et al. (2015), and classification accuracy are reported. For relatedness tasks, we follow Tai et al. (2015) to train a logistic regression to learn the probability distribution of relatedness scores, and we report Pearson's correlation. The four hyper-parameters are chosen the same as those in STS benchmark experiment. For fair comparison, embeddings models are divided into two categories: non-parameterized and parameterized ones, as described in section 1 . Results are shown in Table 3.

GEM outperforms all other non-parameterized sentence embedding models, including SIF, pmean (Rücklé et al., 2018), and BOW on GloVe. The consistent superior performance again demonstrates GEM's advantage on weighting scheme. It also compares favorably with most of parameterized models, including à la carte (Khodak et al., 2018), FastSent (Hill et al., 2016), InferSent, QT, Sent2Vec, SkipThought-LN (with layer normalization) (Kiros et al., 2015), SDAE (Hill et al., 2016), STN (Subramanian et al., 2018) and USE (Yang et al., 2018). Note that sentence representations generated by GEM have much smaller dimension compared to most of benchmark models, and the subsequent neural structure has fewer trainable parameters. This observation suggests that local multi-word level information in sentences has already provided revealing information for sophisticated downstream tasks. The fact that GEM does well on several classification tasks (e.g. TREC and SUBJ) indicates that the proposed weight scheme is able to recognize important words sentences. Also, GEM's competitive performance on sentiment tasks shows that exploiting the geometric structures of two sentence subspaces is semantically informative.

\section{Discussion}

Comparison with Arora et al. (2017). We would like to point out that although sharing the idea of modelling the sentence as the weighted sum of its word vectors, GEM is substantially different from Arora et al. (2017). First, we adopt wellestablished numerical linear algebra to quantify the semantic meaning and importance of words in the sentences context. And this new approach proves to be effective. Second, the weights in SIF (and uSIF) are calculated from the statistic of vocabularies on very large corpus (wikipedia). In contrast, the weights in GEM are directly computed from the sentences themselves along with the dataset, independent with prior statistical knowledge of language or vocabularies. Furthermore, the components in GEM's weights are derived from numerical linear algebra eq. (4) to (9), while SIF directly includes a hyper-parameter term in its weight scheme, i.e. its smooth term.

Robustness and Effectiveness. Besides experiments mentioned above, we also test the robustness and effectiveness GEM on several simple but non-trivial examples. These experiments demonstrate that GEM is quite insensitive against the removal of non-important stop words. Also GEM can correctly assign higher weights for words with more significant semantic meanings in the sentence.

We first test the robustness of GEM by removing one non-important stop word in a sentence and computed the similarity between the original sentence and the one after removal. For example:

- original sentence: "the first tropical system to slam the US this year is expected to make landfall as a hurricane"

- remove 7 stop words: "first tropical system slam US this year expected make landfall hurricane"

The cosine similarity between these two sentences given by GEM is 0.954 . Even though aggressively 


\begin{tabular}{|c|c|c|c|c|c|c|c|c|c|c|c|}
\hline Model & Dim & $\begin{array}{l}\text { Training } \\
\text { time (h) }\end{array}$ & MR & CR & SUBJ & MPQA & SST & TREC & MRPC & SICK-R & SICK-E \\
\hline \multicolumn{12}{|c|}{ Non-parameterized models } \\
\hline GEM + L.F.P (ours) & 900 & 0 & 79.8 & 82.5 & 93.8 & 89.9 & 84.7 & 91.4 & $75.4 / 82.9$ & 86.5 & 86.2 \\
\hline GEM + GloVe (ours) & 300 & 0 & 78.8 & 81.1 & 93.1 & 89.4 & 83.6 & 88.6 & $73.4 / 82.3$ & 86.3 & 85.3 \\
\hline SIF & 300 & 0 & 77.3 & 78.6 & 90.5 & 87.0 & 82.2 & 78.0 & - & 86.0 & 84.6 \\
\hline uSIF & 300 & 0 & - & - & - & - & 80.7 & - & - & 83.8 & 81.1 \\
\hline p-mean & 3600 & 0 & 78.4 & 80.4 & 93.1 & 88.9 & 83.0 & 90.6 & - & - & - \\
\hline GloVe BOW & 300 & 0 & 78.7 & 78.5 & 91.6 & 87.6 & 79.8 & 83.6 & $72.1 / 80.9$ & 80.0 & 78.6 \\
\hline \multicolumn{12}{|l|}{ Paraemterized models } \\
\hline InferSent & 4096 & 24 & 81.1 & 86.3 & 92.4 & 90.2 & 84.6 & 88.2 & $76.2 / 83.1$ & 88.4 & 86.3 \\
\hline Sent2Vec & 700 & 6.5 & 75.8 & 80.3 & 91.1 & 85.9 & - & 86.4 & $72.5 / 80.8$ & - & - \\
\hline SkipThought-LN & 4800 & 336 & 79.4 & 83.1 & 93.7 & 89.3 & 82.9 & 88.4 & - & 85.8 & 79.5 \\
\hline FastSent & 300 & 2 & 70.8 & 78.4 & 88.7 & 80.6 & - & 76.8 & $72.2 / 80.3$ & - & - \\
\hline à la carte & 4800 & N/A & 81.8 & 84.3 & 93.8 & 87.6 & 86.7 & 89.0 & - & - & - \\
\hline SDAE & 2400 & 192 & 74.6 & 78.0 & 90.8 & 86.9 & - & 78.4 & $73.7 / 80.7$ & - & - \\
\hline QT & 4800 & 28 & 82.4 & 86.0 & 94.8 & 90.2 & 87.6 & 92.4 & $76.9 / 84.0$ & 87.4 & - \\
\hline STN & 4096 & 168 & $\underline{82.5}$ & 87.7 & $\overline{94.0}$ & 90.9 & 83.2 & 93.0 & $\underline{78.6 / 84.4}$ & $\underline{88.8}$ & $\underline{87.8}$ \\
\hline USE & 512 & N/A & $\overline{81.36}$ & $\overline{86.08}$ & 93.66 & $\overline{87.14}$ & 86.24 & 96.60 & $\overline{-}$ & $\overline{-}$ & $\overline{-}$ \\
\hline
\end{tabular}

Table 3: Results on supervised tasks. Sentence embeddings are fixed for downstream supervised tasks. Best results for each task are underlined, best results from models in the same category are in bold. SIF results are extracted from Arora et al. (2017) and Rücklé et al. (2018), and training time is collected from Logeswaran and Lee (2018).

removing 7 stop words, GEM still assigns pretty similar embeddings for these two sentences.

We further demonstrate that GEM does assign higher weights to words with more significant semantic meanings. Consider the following sentence: "there are two ducks swimming in the river". Weights assigned by GEM are (sorted from high to low): [ducks: 4.93, river:4.72, swimming: 4.70, two: 3.87 , are: 3.54, there: 3.23 , in:3.04, the:2.93]. GEM successfully assigns higher weight to informative words like ducks and river, and downplay stop words like the and there. More examples can be found in the Appendix.

Ablation Study. As shown in in Table 4, every GEM weight $\left(\alpha_{n}, \alpha_{s}, \alpha_{u}\right)$ and proposed principal components removal methods contribute to the performance. As listed on the left, adding GEM weights improves the score by $8.6 \%$ on STS dataset compared with averaging three concatenated word vectors. The sentence-dependent principal component removal (SDR) proposed in GEM improves $1.7 \%$ compared to directly removing the top $h$ corpus principal components (SIR). Using GEM weights and SDR together yields an overall improvement of $21.1 \%$. As shown on the right in Table 4, every weight contributes to the performance of our model. For example, three weights altogether improve the score in SUBJ task by $0.38 \%$ compared with only using $\alpha_{n}$.

Sensitivity Study. We evaluate the effect of all four hyper-parameters in our model: the win-

\begin{tabular}{ccc}
\hline Configurations & STSB dev & SUBJ \\
\hline Mean of L.F.P & 62.4 & - \\
GEM weights & 71.0 & - \\
GEM weights + SIR & 81.8 & - \\
GEM weights + SDR & 83.5 & - \\
\hline$\alpha_{n}+$ SDR & 81.6 & 93.42 \\
$\alpha_{n}, \alpha_{s}+$ SDR & 81.9 & 93.6 \\
$\alpha_{n}, \alpha_{s}, \alpha_{u}+$ SDR & 83.5 & 93.8 \\
\hline
\end{tabular}

Table 4: Comparison of different configurations demonstrates the effectiveness of our model on STSB dev set and SUBJ. SDR stands for sentence-dependent principal component removal in Section 2.4.2. SIR stands for sentence-independent principal component removal, i.e. directly removing top $h$ corpus principal components from the sentence embedding.

dow size $m$ in the contextual window matrix, the number of candidate principal components $K$, the number of principal components to remove $h$, and the power of the singular value in coarse sentence embedding, i.e. the power $t$ in $f\left(\sigma_{j}\right)=\sigma_{j}^{t}$ in Equation (7). We sweep the hyper-parameters and test on STSB dev set, SUBJ, and MPQA. Unspecified parameters are fixed at $m=7, K=45$, $h=17$ and $t=3$. As shown in Figure 2, our model is quite robust with respect to hyperparameters.

Inference speed. We also compare the inference speed of our algorithm on the STSB test set 

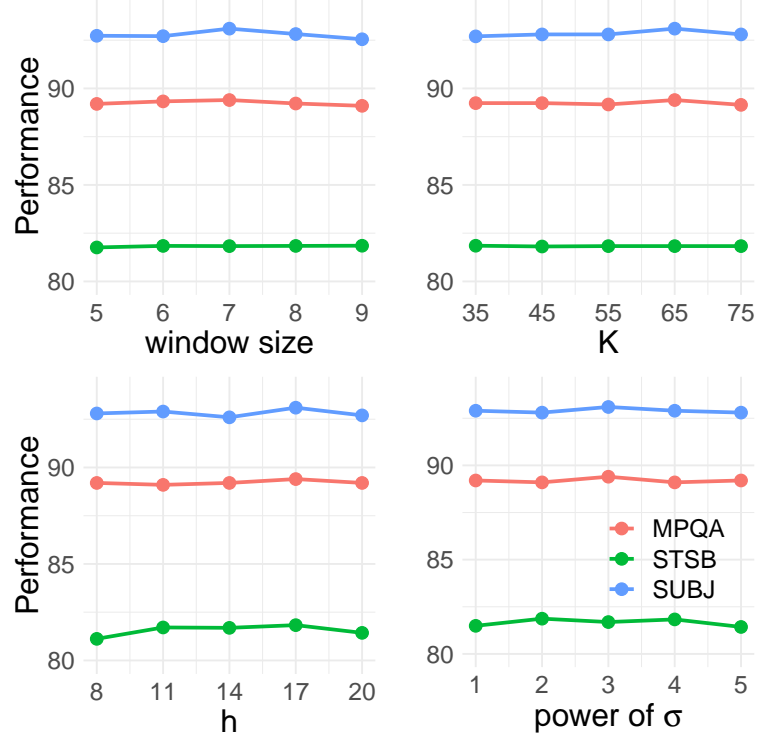

Figure 2: Sensitivity tests on four hyper-parameters, the window size $m$ in contextual window matrix, the number of candidate principal components $K$, the number of principal components to remove $h$, and the exponential power of singular value in coarse sentence embedding.

with the benchmark models SkipThought and InferSent. SkipThought and InferSent are run on a NVIDIA Tesla P100 GPU, and our model is run on a CPU (Intel Xeon CPU E5-2690 v4 @2.60GHz). For fair comparison, batch size in InferSent and SkipThought is set to be 1 . The results are shown in Table 5. It shows that without acceleration from GPU, our model is still faster than InferSent and is $54 \%$ faster than SkipThought.

\begin{tabular}{ccc}
\hline & Average run time (s) & Variance \\
\hline GEM (CPU) & $\mathbf{2 0 . 0 8}$ & 0.23 \\
InferSent(GPU) & 21.24 & 0.15 \\
SkipThought (GPU) & 43.36 & 0.10 \\
\hline
\end{tabular}

Table 5: Run time of GEM, InferSent and SkipThought on encoding sentences in STSB test set. GEM is run on CPU, InferSent and SkipThought is run on GPU. Data are collected from 5 trials.

\section{Conclusions}

We proposed a simple non-parameterized method to generate sentence embeddings, based entirely on the geometric structure of the subspace spanned by word embeddings. Our sentence embedding evolves from the new orthogonal basis vector brought in by each word, which represents novel semantic meaning. The evaluation shows that our method not only sets up the new state-of-the-art of non-parameterized models but also performs competitively when compared with models requiring either large amount of training data or prolonged training time. In future work, we plan to consider subwords into the model and explore more geometric structures in sentences.

\section{Acknowledgments}

We would like to thank Jade Huang for proofreading the paper and helpful writing suggestions. We also acknowledge the anonymous reviewers for their valuable feedback.

\section{References}

Sanjeev Arora, Yingyu Liang, and Tengyu Ma. 2017. A simple but tough-to-beat baseline for sentence embeddings. International Conference on Learning Representations.

Steven Bird, Ewan Klein, and Edward Loper. 2009. Natural language processing with Python: analyzing text with the natural language toolkit. "O'Reilly Media, Inc.".

Daniel Cer, Mona Diab, Eneko Agirre, Inigo LopezGazpio, and Lucia Specia. 2017. Semeval-2017 task 1: Semantic textual similarity-multilingual and cross-lingual focused evaluation. arXiv preprint arXiv:1708.00055.

Daniel Cer, Yinfei Yang, Sheng-yi Kong, Nan Hua, Nicole Limtiaco, Rhomni St John, Noah Constant, Mario Guajardo-Cespedes, Steve Yuan, Chris Tar, et al. 2018. Universal sentence encoder. arXiv preprint arXiv:1803.11175.

Delphine Charlet and Geraldine Damnati. 2017. Simbow at semeval-2017 task 3: Soft-cosine semantic similarity between questions for community question answering. In Proceedings of the 11th International Workshop on Semantic Evaluation (SemEval2017), pages 315-319.

Alexis Conneau, Douwe Kiela, Holger Schwenk, Loïc Barrault, and Antoine Bordes. 2017. Supervised learning of universal sentence representations from natural language inference data. In Proceedings of the 2017 Conference on Empirical Methods in Natural Language Processing, pages 670-680, Copenhagen, Denmark. Association for Computational Linguistics.

Jacob Devlin, Ming-Wei Chang, Kenton Lee, and Kristina Toutanova. 2018. Bert: Pre-training of deep bidirectional transformers for language understanding. arXiv preprint arXiv:1810.04805. 
Bill Dolan, Chris Quirk, and Chris Brockett. 2004. Unsupervised construction of large paraphrase corpora: Exploiting massively parallel news sources. In Proceedings of the 20th international conference on Computational Linguistics, page 350. Association for Computational Linguistics.

Kawin Ethayarajh. 2018. Unsupervised random walk sentence embeddings: A strong but simple baseline. In Proceedings of The Third Workshop on Representation Learning for NLP, pages 91-100.

Simone Filice, Giovanni Da San Martino, and Alessandro Moschitti. 2017. Kelp at semeval-2017 task 3: Learning pairwise patterns in community question answering. In Proceedings of the 11th International Workshop on Semantic Evaluation (SemEval-2017), pages 326-333.

Felix Hill, Kyunghyun Cho, and Anna Korhonen. 2016. Learning distributed representations of sentences from unlabelled data. arXiv preprint arXiv:1602.03483.

Minqing $\mathrm{Hu}$ and Bing Liu. 2004. Mining and summarizing customer reviews. In Proceedings of the tenth ACM SIGKDD international conference on Knowledge discovery and data mining, pages 168-177. ACM.

Mikhail Khodak, Nikunj Saunshi, Yingyu Liang, Tengyu Ma, Brandon Stewart, and Sanjeev Arora. 2018. A la carte embedding: Cheap but effective induction of semantic feature vectors. arXiv preprint arXiv: 1805.05388 .

Ryan Kiros, Yukun Zhu, Ruslan R Salakhutdinov, Richard Zemel, Raquel Urtasun, Antonio Torralba, and Sanja Fidler. 2015. Skip-thought vectors. In Advances in neural information processing systems, pages 3294-3302.

Lajanugen Logeswaran and Honglak Lee. 2018. An efficient framework for learning sentence representations. International Conference on Learning Representations.

Marco Marelli, Luisa Bentivogli, Marco Baroni, Raffaella Bernardi, Stefano Menini, and Roberto Zamparelli. 2014. Semeval-2014 task 1: Evaluation of compositional distributional semantic models on full sentences through semantic relatedness and textual entailment. In Proceedings of the 8th international workshop on semantic evaluation (SemEval 2014), pages $1-8$.

Tomas Mikolov, Ilya Sutskever, Kai Chen, Greg S Corrado, and Jeff Dean. 2013. Distributed representations of words and phrases and their compositionality. In Advances in neural information processing systems, pages 3111-3119.

Matteo Pagliardini, Prakhar Gupta, and Martin Jaggi. 2018. Unsupervised Learning of Sentence Embeddings using Compositional n-Gram Features. In NAACL 2018 - Conference of the North American
Chapter of the Association for Computational Linguistics.

Bo Pang and Lillian Lee. 2004. A sentimental education: Sentiment analysis using subjectivity summarization based on minimum cuts. In Proceedings of the 42nd annual meeting on Association for Computational Linguistics, page 271. Association for Computational Linguistics.

Bo Pang and Lillian Lee. 2005. Seeing stars: Exploiting class relationships for sentiment categorization with respect to rating scales. In Proceedings of the 43rd annual meeting on association for computational linguistics, pages 115-124. Association for Computational Linguistics.

Jeffrey Pennington, Richard Socher, and Christopher Manning. 2014. Glove: Global vectors for word representation. In Proceedings of the 2014 conference on empirical methods in natural language processing (EMNLP), pages 1532-1543.

A. Rücklé, S. Eger, M. Peyrard, and I. Gurevych. 2018. Concatenated $p$-mean Word Embeddings as Universal Cross-Lingual Sentence Representations. ArXiv e-prints.

Alexandre Salle, Aline Villavicencio, and Marco Idiart. 2016. Matrix factorization using window sampling and negative sampling for improved word representations. CoRR, abs/1606.00819.

Richard Socher, Alex Perelygin, Jean Wu, Jason Chuang, Christopher D Manning, Andrew Ng, and Christopher Potts. 2013. Recursive deep models for semantic compositionality over a sentiment treebank. In Proceedings of the 2013 conference on empirical methods in natural language processing, pages 1631-1642.

Karen Sparck Jones. 1972. A statistical interpretation of term specificity and its application in retrieval. Journal of documentation, 28(1):11-21.

Sandeep Subramanian, Adam Trischler, Yoshua Bengio, and Christopher J Pal. 2018. Learning general purpose distributed sentence representations via large scale multi-task learning. arXiv preprint arXiv:1804.00079.

Kai Sheng Tai, Richard Socher, and Christopher D Manning. 2015. Improved semantic representations from tree-structured long short-term memory networks. arXiv preprint arXiv:1503.00075.

Ashish Vaswani, Noam Shazeer, Niki Parmar, Jakob Uszkoreit, Llion Jones, Aidan N Gomez, Łukasz Kaiser, and Illia Polosukhin. 2017. Attention is all you need. In Advances in Neural Information Processing Systems, pages 5998-6008.

Ellen M Voorhees and Hoa Trang Dang. 2003. Overview of the trec 2003 question answering track. In TREC, volume 2003, pages 54-68. 
Janyce Wiebe, Theresa Wilson, and Claire Cardie. 2005. Annotating expressions of opinions and emotions in language. Language resources and evaluation, 39(2-3):165-210.

John Wieting, Mohit Bansal, Kevin Gimpel, and Karen Livescu. 2015a. Towards universal paraphrastic sentence embeddings. In International Conference on Learning Representation.

John Wieting, Mohit Bansal, Kevin Gimpel, Karen Livescu, and Dan Roth. 2015b. From paraphrase database to compositional paraphrase model and back. arXiv preprint arXiv:1506.03487.

John Wieting and Kevin Gimpel. 2017a. Paranmt-50m: Pushing the limits of paraphrastic sentence embeddings with millions of machine translations. arXiv preprint arXiv:1711.05732.

John Wieting and Kevin Gimpel. 2017b. Revisiting recurrent networks for paraphrastic sentence embeddings. In Proceedings of the Annual Meeting of the Association for Computational Linguistics.

Y. Yang, S. Yuan, D. Cer, S.-y. Kong, N. Constant, P. Pilar, H. Ge, Y.-H. Sung, B. Strope, and R. Kurzweil. 2018. Learning Semantic Textual Similarity from Conversations. ArXiv e-prints. 


\section{A Robustness and Effectiveness of GEM}

\section{A.1 Robustness Test}

We test the robustness of GEM by removing one non-important stop word in a sentence and computed the similarity between the original sentence and the one after removal. For example:

- original $=$ "The student is reading a physics book"

- removed = "student is reading a physics book"

Stop word "The" is removed. The cosine similarity between embeddings of the two sentences generated by GEM is 0.998 . GEM assigns pretty similar embeddings for these two sentences even with the removal of stop words, especially this is a short sentence with only 7 words. More examples are:

- original $=$ "Someone is sitting on the blanket"

- removed = "Someone is sitting on blanket"

- cosine similarity $=0.981$

and

- original $=" A$ man walks along walkway to the store"

- removed = "man walks along walkway to the store"

- cosine similarity $=0.984$

These experiments prove that GEM is robust against stop words and words order.

\section{A.2 Effectiveness Test}

We also demonstrate that GEM assign higher weights to words with more significant meanings. Consider the sentence: the stock market closes lower on Friday, weights assigned by GEM are [lower: 4.94, stock: 4.93, closes: 4.78, market: 4.62, Friday: 4.51, the: 3.75, on: 3.70]. Again, GEM emphasizes informative words like lower and closes, and diminishes stop words like the and there.

\section{B Proof}

The novelty score $\left(\alpha_{n}\right)$, significance score $\left(\alpha_{s}\right)$ and corpus-wise uniqueness score $\left(\alpha_{u}\right)$ are larger when a word $w$ has relatively rare appearance in the corpus and can bring in new and important semantic meaning to the sentence.

Following the section 3 in Arora et al. (2017), we can use the probability of a word $w$ emitted from sentence $s$ in a dynamic process to explain eq. (10) and put this as following Theorem with its proof provided below.

Theorem 1. Suppose the probability that word $w_{i}$ is emitted from sentence $s$ is ${ }^{2}$ :

$\mathrm{p}\left[w_{i} \mid \boldsymbol{c}_{s}\right] \propto\left(\frac{\exp \left(\left\langle\boldsymbol{c}_{s}, \boldsymbol{v}_{w_{i}}\right\rangle\right)}{Z}+\exp \left(-\left(\alpha_{n}+\alpha_{s}+\alpha_{u}\right)\right)\right)$

where $c_{s}$ is the sentence embedding, $Z=$ $\sum_{w_{i} \in \mathcal{V}} \exp \left(\left\langle\boldsymbol{c}_{s}, \boldsymbol{v}_{w_{i}}\right\rangle\right)$ and $\mathcal{V}$ denotes the vocabulary. Then when $Z$ is sufficiently large, the MLE for $\boldsymbol{c}_{s}$ is:

$$
\boldsymbol{c}_{s} \propto \sum_{w_{i} \in s}\left(\alpha_{n}+\alpha_{s}+\alpha_{u}\right) \boldsymbol{v}_{w_{i}}
$$

Proof: According to Equation (12),

$\mathrm{p}\left[w_{i} \mid \boldsymbol{c}_{s}\right]=\frac{1}{N}\left(\frac{\exp \left(\left\langle\boldsymbol{c}_{s}, \boldsymbol{v}_{w_{i}}\right\rangle\right)}{Z}+\exp \left(-\left(\alpha_{n}+\alpha_{s}+\alpha_{u}\right)\right)\right)$

Where $N$ and $Z$ are two partition functions defined as

$$
\begin{gathered}
N=1+\sum_{w_{i} \in \mathcal{V}} \exp \left(-\left(\alpha_{n}\left(w_{i}\right)+\alpha_{s}\left(w_{i}\right)+\alpha_{u}\left(w_{i}\right)\right)\right) \\
Z=\sum_{w_{i} \in \mathcal{V}} \exp \left(\left\langle\boldsymbol{c}_{s}, \boldsymbol{v}_{w_{i}}\right\rangle\right)
\end{gathered}
$$

The joint probability of sentence $s$ is then

$$
p\left(s \mid \boldsymbol{c}_{s}\right)=\prod_{w_{i} \in s} p\left(w_{i} \mid \boldsymbol{c}_{s}\right)
$$

To simplify the notation, let $\alpha=\alpha_{n}+\alpha_{s}+\alpha_{u}$. It follows that the log likelihood $f\left(w_{i}\right)$ of word $w_{i}$ emitted from sentence $s$ is given by

$$
f_{w_{i}}\left(\boldsymbol{c}_{s}\right)=\log \left(\frac{\exp \left(\left\langle\boldsymbol{c}_{s}, \boldsymbol{v}_{w_{i}}\right\rangle\right)}{Z}+e^{-\alpha}\right)-\log (N)
$$

\footnotetext{
${ }^{2}$ The first term is adapted from Arora et al. (2017), where words near the sentence vector $c_{s}$ has higher probability to be generated. The second term is introduced so that words similar to the context in the sentence or close to common words in the corpus are also likely to occur.
} 


$$
\nabla f_{w_{i}}\left(\boldsymbol{c}_{s}\right)=\frac{\exp \left(\left\langle c_{s}, \boldsymbol{v}_{w_{i}}\right\rangle\right) \boldsymbol{v}_{w_{i}}}{\exp \left(\left\langle\boldsymbol{c}_{s}, \boldsymbol{v}_{w_{i}}\right\rangle\right)+Z e^{-\alpha}}
$$

By Taylor expansion, we have

$$
\begin{aligned}
f_{w_{i}}\left(\boldsymbol{c}_{s}\right) & \approx f_{w_{i}}(0)+\nabla f_{w_{i}}(0)^{T} \boldsymbol{c}_{s} \\
& =\text { constant }+\frac{\left\langle\boldsymbol{c}_{s}, \boldsymbol{v}_{w_{i}}\right\rangle}{Z e^{-\alpha}+1}
\end{aligned}
$$

Again by Taylor expansion on $Z$,

$$
\begin{aligned}
\frac{1}{Z e^{-\alpha}+1} & \approx \frac{1}{1+Z}+\frac{Z}{(1+Z)^{2}} \alpha \\
& \approx \frac{Z}{(1+Z)^{2}} \alpha \\
& \approx \frac{1}{1+Z} \alpha
\end{aligned}
$$

The approximation is based on the assumption that $Z$ is sufficiently large. It follows that,

$$
f_{w_{i}}\left(\boldsymbol{c}_{s}\right) \approx \text { constant }+\frac{\alpha}{1+Z}\left\langle\boldsymbol{c}_{s}, \boldsymbol{v}_{w_{i}}\right\rangle
$$

Then the maximum log likelihood estimation of $c_{s}$ is:

$$
\begin{aligned}
\boldsymbol{c}_{s} & \approx \sum_{w_{i} \in s} \frac{\alpha}{1+Z} \boldsymbol{v}_{w_{i}} \\
& \propto \sum_{w_{i} \in s}\left(\alpha_{n}+\alpha_{s}+\alpha_{u}\right) \boldsymbol{v}_{w_{i}}
\end{aligned}
$$

\section{Experimental settings}

For all experiments, sentences are tokenized using the NLTK tokenizer (Bird et al., 2009) wordpunct_tokenize, and all punctuation is skipped. $f\left(\sigma_{j}\right)=\sigma_{j}^{t}$ in Equation (7). In the STS benchmark dataset, our hyper-parameters are chosen by conducting parameters search on STSB dev set at $m=7, h=17, K=45$, and $t=3$. And we use the same values for all supervised tasks. The integer interval of parameters search are $m \in[5,9]$, $h \in[8,20], L \in[35,75]$ (at stride of 5), and $t \in[1,5]$. In CQA dataset, $m$ and $h$ are changed to 6 and 15, the correlation term in section 2.4 .2 is changed to $o_{i}=\left\|\boldsymbol{S}^{T} \boldsymbol{d}_{i}\right\|_{2}$ empirically. In supervised tasks, same as Arora et al. (2017), we do not perform principal components in supervised tasks.

\section{Clarifications on Linear Algebra}

\section{D.1 Encode a long sequence of words}

We would like to give a clarification on encoding a long sequence of words, for example, a paragraph or a article. Specifically, the length $n$ of the sequence is larger than the dimension $d$ of pretrained word vectors in this case. The only part in GEM relevant to the length of the sequence $n$ is the coarse embedding in Equation (7). The SVD of the sentence matrix of the $i$ th sentence is still $\boldsymbol{S} \in \mathbb{R}^{d \times n}=\left[\boldsymbol{v}_{w_{1}}, \ldots, \boldsymbol{v}_{w_{n}}\right]=\boldsymbol{U} \boldsymbol{\Sigma} \boldsymbol{V}^{T}$, where now $\boldsymbol{U} \in \mathbb{R}^{d \times d}, \boldsymbol{\Sigma} \in \mathbb{R}^{d \times n}$, and $\boldsymbol{V} \in$ $R^{n \times n}$. Note that the $d+1$ th column to $n$th column in $\Sigma$ are all zero. And Equation (7) becomes $\boldsymbol{g}_{i}=\sum_{j=1}^{d} f\left(\sigma_{j}\right) \boldsymbol{U}_{:, j}$. The rest of the algorithm works as usual. Also, Gram-Schmidt (GS) process is computed in the context window of word $w_{i}$, and the length of context window is set to be $2 m+1=17$ in STS benchmark dataset and supervise downstream tasks. That is, GS is computed on 17 vectors, and 17 is smaller than the dimension $d$. Therefore, GS is always validate in our model, independent with the length of the sentence.

\section{D.2 Sensitivity to Word Order}

Although utilizing Gram-Schmidt process (GS), GEM is insensitive to the order of words in the sentence, explained as follows. The new semantic meaning vector $q_{i}$ computed from doing GS on the context window matrix $S^{i}$ is independent with the relative order of first $2 m$ vectors. This is because in GEM $w_{i}$ (the word we are calculating weights for) is always shifted to be the last column of $\boldsymbol{S}^{i}$. And weighting scheme in GEM only depends on $\boldsymbol{q}_{i}$. Therefore, weight scores stay the same for $w_{i}$. 